\title{
Exploring the Relationship between Disease Awareness and Outcomes in Patients with Chronic Obstructive Pulmonary Disease
}

\author{
Ilaria Baiardini ${ }^{a}$ Marco Contoli ${ }^{b}$ Angelo Guido Corsico ${ }^{c}$ Carla Scognamillo $^{d}$ \\ Fabio Ferri $^{\mathrm{e}}$ Nicola Scichilone ${ }^{f}$ Paola Rogliani ${ }^{g}$ Fabiano Di Marco ${ }^{\mathrm{h}}$ \\ Pierachille Santus ${ }^{i}$ Fulvio Braido ${ }^{a}$
}

\begin{abstract}
aRespiratory Unit for Continuity of Care, Department of Internal Medicine, University of Genova, Genoa, Italy; ${ }^{b}$ Respiratory Diseases Department of Morphology, Surgery and Experimental Medicine-Università di FerraraFerrara, Ferrara, Italy; ${ }^{~}$ Respiratory Diseases Division, Medical Sciences and Infectious Diseases Department, IRCCS Policlinico San Matteo Foundation, and Department of Internal Medicine and Therapeutics, University of Pavia, Pavia, Italy; ${ }^{\mathrm{d} B o e h r i n g e r ~ I n g e l h e i m, ~ M i l a n, ~ I t a l y ; ~}{ }^{\mathrm{e}}$ MediNeos Observational Research, Modena, Italy; ${ }^{\mathrm{f} D i p a r t i m e n t o}$ Universitario PROMISE, Università di Palermo, UOC di Pneumologia, Policlinico Universitario P. Giaccone, Palermo, Italy; ${ }^{9}$ Respiratory Medicine, Department of Experimental Medicine Università di Roma Tor Vergata,", Rome, Italy; h'Department of Health Sciences, Università degli Studi di Milano, Respiratory Unit, ASST Papa Giovanni XXIII, Bergamo, Italy; 'Department of Biomedical and Clinical Sciences (DIBIC), Università degli Studi di Milano. Division of Respiratory Diseases, University Hospital "L. Sacco," ASST Fatebenefratelli-Sacco, Milan, Italy
\end{abstract}

\section{Keywords}

Awareness · Chronic obstructive pulmonary disease ·

Adherence $\cdot$ Patient-reported outcomes

\begin{abstract}
Background: Disease awareness is a challenge in the management of chronic obstructive pulmonary disease (COPD). Objectives: The aim of this analysis was to explore the association between COPD optimal and suboptimal awareness, clinical parameters, and the following patient-reported outcomes: modified Medical Research Council (mMRC), Treatment Satisfaction Questionnaire (TSQM-9), COPD Assessment Test (CAT), Morisky Medication-Taking Adherence Scale (MMAS-4), and Brief Illness Perception Questionnaire (B-IPQ). Methods: This post hoc analysis of the SAT study included all enrolled patients for whom awareness (Disease Awareness in COPD Questionnaire - DACQ) was assessed at baseline and 12 months. DACQ scores $\geq 80$ were considered
\end{abstract}

karger@karger.com www.karger.com/res

Karger $\stackrel{\text { ' }}{5}$

GOPEN ACCESS
(C) 2021 The Author(s)

Published by S. Karger AG, Basel

This is an Open Access article licensed under the Creative Commons Attribution-NonCommercial-4.0 International License (CC BY-NC) (http://www.karger.com/Services/OpenAccessLicense), applicable to the online version of the article only. Usage and distribution for commercial purposes requires written permission. an indicator of an optimal awareness. Results: 367 patients (25.8\% women, median age 72 years) were included in the analysis. At enrollment, 74 patients $(20.2 \%)$ had a DACQ score $\geq 80$. Patients with suboptimal awareness, compared to those in which awareness was optimal, had higher median scores for CAT $(p=0.0001)$ and mMRC $(p=0.0031)$, a lower median TSQM-9 global score $(p<0.0001)$, and higher median B-IPQ score $(p<0.0001)$. The proportion of patients who had exacerbations during the previous year was higher in patients with suboptimal COPD awareness than in those with DACQ score $\geq 80$ ( 42.8 vs. $21.4 \%, p=0.0009$ ). During the 12 -month observation period, illness perception, adherence, and treatment satisfaction were found to be independent factors significantly associated with level of disease awareness. Conclusion: The results of our post hoc analysis suggest that patients' awareness of their COPD disease is related to both clinical outcomes and how they perceive and manage their condition.

(c) 2021 The Author(s)

Published by S. Karger AG, Basel
Ilaria Baiardini

Department of Internal Medicine, University of Genova

Azienda Policlinico IRCCS San Martino

Viale Benedetto XV, 5, IT-16132 Genoa (Italy)

ilaria.baiardini@libero.it 


\section{Introduction}

Improved disease awareness has been identified in recent years as one of the major public health and medical challenges for the detection and management of chronic obstructive pulmonary disease (COPD) because of the potential impact on the morbidity and mortality associated with this condition $[1,2]$. Major efforts to increase public knowledge about COPD prevalence, symptoms, diagnosis, and treatment have been undertaken over the past decades, by means of campaigns, education and advocacy programs, and activities in more than 50 countries worldwide [3-5]. However, recent studies show that COPD remains highly underrepresented in the public interest [6] and among subjects who are at high risk of developing the disease $[7,8]$. Furthermore, the low awareness of the disease is considered the first factor that limits early COPD management [9].

Being informed about the characteristics, consequences, and treatment of COPD is a necessary step toward improving disease management. However, even when patients have a good overall knowledge of their health condition, they may have difficulties in understanding, accepting, and managing it. This happens because the issue is more complex than to be informed [10]. Awareness has been defined as "a reasonable or realistic perception or appraisal of one's situation, functioning or performance, or of the resulting implications, expressed explicitly or implicitly" [11]; it depends not only on the correctness of information but refers to a complex process in which patients assign a personal meaning to their knowledge and integrates feelings and cognition into their disease experience [12]. The Disease Awareness in COPD Questionnaire (DACQ) has been recently validated to assess patients' awareness of COPD [13] by exploring different dimensions: the mastery of a correct knowledge on COPD; the level of subjective view on necessity and role of COPD therapy; the patient perceptions of COPD in terms of features and consequences; and the individual acceptance of thoughts and feelings related to COPD.

The DACQ has been developed and tested in the context of the "SATisfaction and adherence to COPD treatment" (SAT) study [14] and demonstrated to be a reliable and valid tool. The analysis of the DACQ scores highlighted that, despite the fact that patients were diagnosed and cared for in specialist COPD settings, the level of awareness was suboptimal. Using the data of the SAT study, we explored the association between COPD optimal and suboptimal awareness, clinical parameters, and patient-reported outcomes.

\section{Material and Methods}

Study Design

This is a post hoc analysis of the SAT study (ClinicalTrials.gov number NCT02689492). The methodology and results from the SAT study have been reported previously [12]. In brief, the SAT study was a multicenter, observational perspective cohort study. 401 consecutive COPD patients, referred to specialist centers for a follow-up visit, were enrolled over a period of approximately 8 months. Patients were followed up for 1 year, with an intermediate evaluation after $6(+/-1)$ months from enrollment (compatible with current clinical practice in Italy for COPD patient management), from November 2015 to October 2017.

At each visit, patients filled the DACQ along with the following validated questionnaires (Italian versions):

- The Treatment Satisfaction Questionnaire for Medication (TSQM-9) [15, 16], a 9-item tool aimed to evaluate the satisfaction with pharmacological treatment. The TSQM-9 provides scores on 3 scales: effectiveness, convenience, and global satisfaction, the higher the score and the higher the level of satisfaction.

- The Brief Illness Perception Questionnaire (B-IPQ) [17], a 9-item questionnaire designed to rapidly assess cognitive and emotional representation of illness. The total score ranges from 8 to 80 : a higher score reflects a more threatening view of the illness.

- The Morisky Medication-Taking Adherence Scale (MMAS-4) [18], a self-reported, medication-taking behavior scale. It consists of 4 questions about the way patients might experience drug errors or omissions. The total score ranges from 0 to 4 ; a higher score indicates better adherence to therapy.

- The COPD Assessment Test (CAT) [19], an 8-item unidimensional measure of health status impairment in COPD. The scores range from 0 to 40; higher scores represent worse health.

- The Modified Medical Research Council (mMRC) scale [20], a widely used tool for grading the effect of breathlessness on daily activities [21]. The physician asks the patient about his/her perceived breathlessness and uses the modified MRC dyspnea scale to classify it from 0 (breathless with strenuous exercise) to 4 (too breathless to leave the house or breathless when dressing or undressing).

For TSQM-9, B-IPQ, and MMAS-4 scales, the authors reported no validated cutoff values for minimal clinically important difference [22], defined as the smallest change in an outcome measure perceived as beneficial by patients. This post hoc analysis included all evaluable enrolled patients who filled in the DACQ at enrollment and at 12-month follow-up. The level of awareness for each item is evaluated on a four-point scale: optimal (4), suboptimal (3), scarce (2), or absent (1). Domain scores are normalized to a scale ranging from 0 to 100 with higher scores corresponding to a better level of awareness. Similar to other PROs for which scores of $80 \%$ or higher are reflective of the achievement of the outcome [23, 24], normalized scores $\geq 80$ have been considered an indicator of an optimal level of awareness.

The analyzed endpoints were (1) the percentage of patients' who achieved an optimal level of COPD awareness at enrollment; (2) the differences in terms of clinical characteristics and PROs between patients with and without optimal awareness of COPD; (3) the rate of exacerbation in the previous year and the CAT scores in patients with optimal and suboptimal awareness; and (4) the relationship between DACQ total score and clinical characteristics/PROs during the 12-month observation period. 
Table 1. Demographics, clinical characteristics, and patient-reported outcomes of patients at enrollment

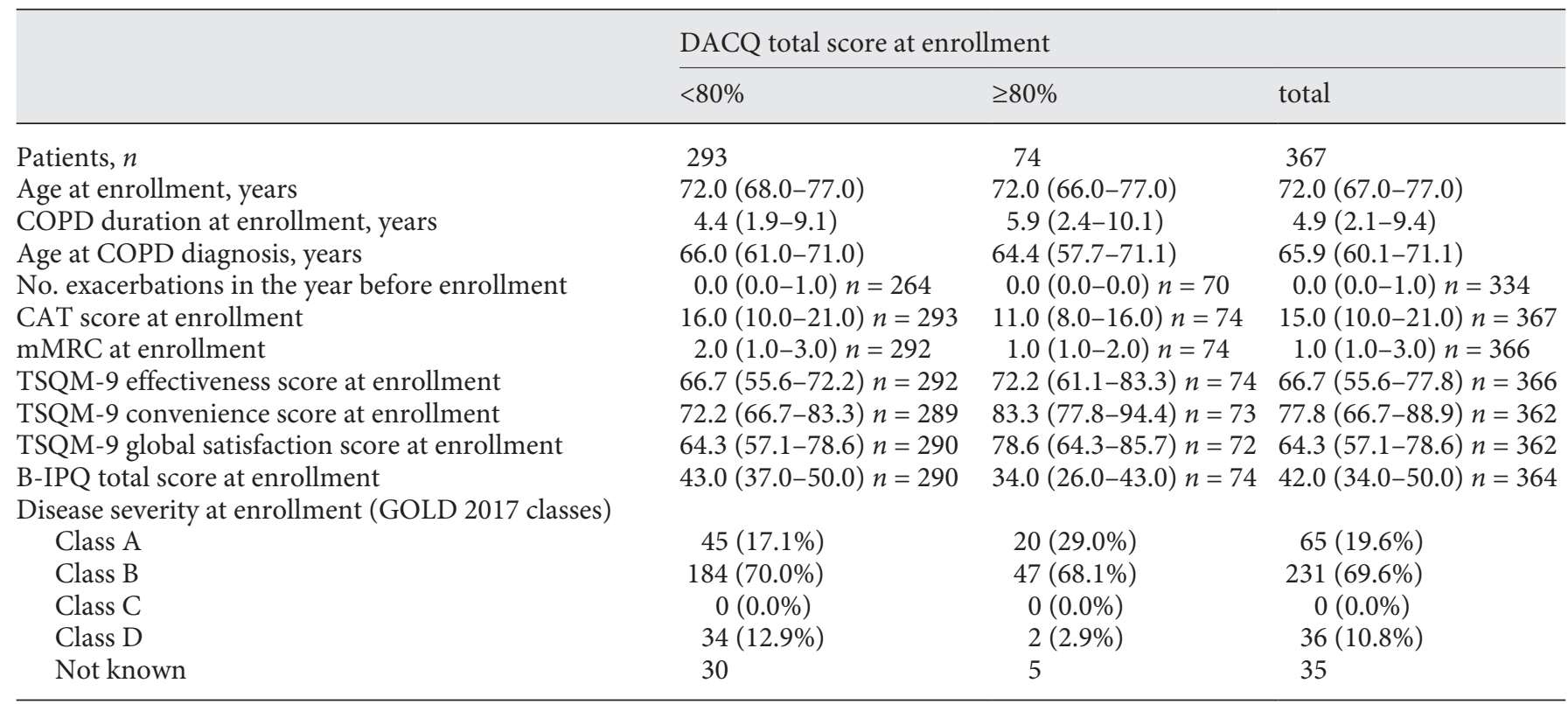

For numeric variables, the median (with 25th-75th percentiles) was shown. For categorical variables, percentages were calculated by column (considering the number of patients with available data as denominator). When the number of patients with available data for specific variables was lower than the number of patients specified in the table headings, this was specified.

\section{Statistical Analysis}

Descriptive statistics were used to evaluate sociodemographic and clinical variables and PROs at the enrollment visit and at final study visit. For numeric variables, median values (with interquartile range) were calculated. Categorical variables were reported as absolute and relative frequencies. Two-sample $t$-test (or respective analogous nonparametric tests, when appropriate) was used to evaluate the statistical significance of differences in numeric variables between different groups. Fisher exact test was used to assess the association between categorical variables. Repeated measurement linear regression models were used to calculate the regression $\beta$ coefficients for (i) the evaluation of the associations between level of COPD awareness (dependent variable) and the independent variables such as clinical characteristics (number of annual exacerbations, mMRC dyspnoea grade) and patient-reported outcomes (B-IPQ total score, MMMS-4 score, and TSQM-9 scores) at enrollment and at 12-month follow-up visits and (ii) the evaluation of the associations between CAT score (dependent variable) and independent variables such as clinical characteristics or PROs during the 12-month observation period. The statistical analysis was performed with SAS ${ }^{\circledR} 9.4$ software.

\section{Results}

A total of 401 patients were enrolled in the SAT study; 367 of them (25.8\% women) were eligible for inclusion in the post hoc analysis since DACQ were incomplete or missing for 34 patients at enrollment visit. Demographics, clinical characteristics, and PROs at enrollment are reported in Table 1.

- Percentage of patients' who achieved a good level of COPD awareness at the enrollment. At enrollment, an optimal level of awareness (DACQ total score $\geq 80$ ) was identified in 74 patients (20.2\%), whereas 293 patients $(78.8 \%)$ did not achieve this level.

- Between-group differences in socio-demographics, clinical characteristics and in PROs according to level of awareness. At enrollment, no difference between the 2 groups was found in terms of age, age at COPD diagnosis, and MMAS scores. Overall, patients with suboptimal awareness, compared to those in which awareness was optimal, had a higher median CAT score (difference: 5.0 points; $p$ value $=0.0001)$, a higher median mMRC score (difference: 1.0 point; $p$ value $=0.0031$ ), a lower median duration of COPD (difference: 1.4 years; $p$ value $=0.0428$ ), a lower median TSQM-9 effectiveness score (difference: 5.6 points; $p$ value $=$ $0.0002)$, a lower median TSQM-9 convenience score (difference: 11.1 points; $p$ value $<0.0001$ ), a lower median TSQM-9 global satisfaction score (difference: 14.3 points; $p$ value $<0.0001$ ), and a higher median B-IPQ total score (difference: 9.0 points; $p$ value $<0.0001$ ). 


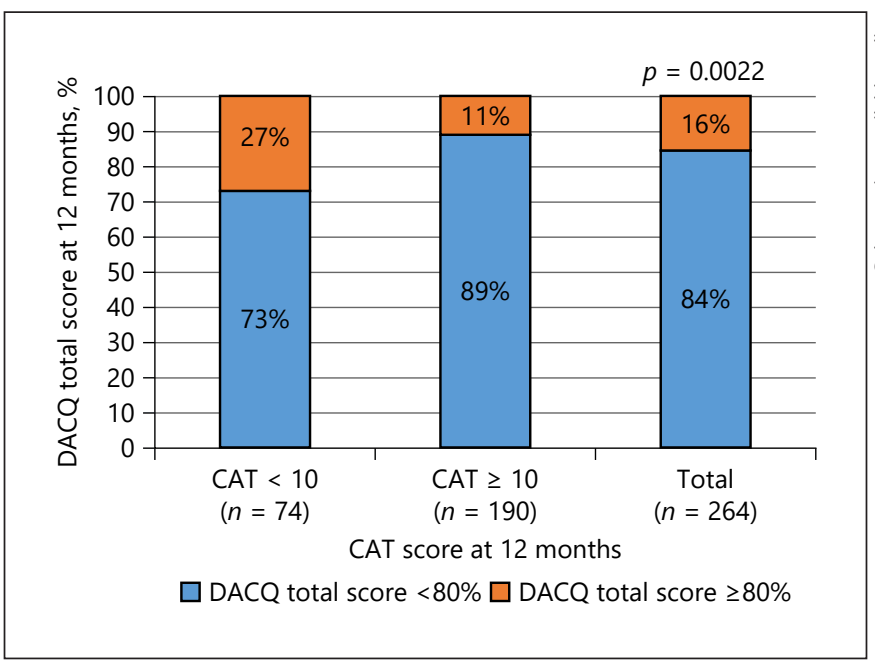

Fig. 1. Difference in the proportion of patients with an optimal awareness (DACQ total score $\geq 80$ ) in the group with CAT $<10$ and in the group of with CAT $\geq 10$ at the 12 -month follow-up ( $p=$ 0.0022).

- Exacerbation history and CAT scores in patients with optimal and suboptimal awareness. At enrollment, the proportion of patients who had exacerbations during the previous year was higher in patients with suboptimal COPD awareness (DACQ total score $<80$ ) than in those with an optimal level of awareness (42.8 vs. $21.4 \%, p$ value $=0.0009$ ). At the 12 -month follow-up, the proportion of patients with an optimal awareness (DACQ total score $\geq 80$ ) was significantly higher in the group of those with CAT $<10$ than in the group of those with CAT $\geq 10$ ( $p=0.0022)$ (Fig. 1).

- Relationship between COPD awareness and clinical characteristics/PROs during the 12-month observation period: a repeated measures regression model was implemented to assess the relationship between DACQ total score and clinical parameters. In particular, TSQM-9 scores, B-IPQ score, level of adherence (MMAS-4 score), and number of annual exacerbations were considered as independent variables; CAT and $\mathrm{mMRC}$ scores were not included as covariates in the model because they were both highly correlated to B-IPQ score (Spearman's correlation coefficients $>0.65$ and $>0.55$, respectively; $p<0.0001$ in both cases). The following independent factors were found to be significantly associated with level of awareness: illness perception, adherence, and treatment satisfaction (Table 2). The relationship between CAT and awareness, as measured at enrollment and at 12-month follow-up visits, was evaluated by means of another repeated
Table 2. Relationship between DACQ total score and PROs over the 12-month observation period (repeated measures regression model for numeric variables)

\begin{tabular}{lclr} 
Effect & $\begin{array}{l}\text { Estimate } \\
(\beta)\end{array}$ & $\begin{array}{l}\text { Standard } \\
\text { error }\end{array}$ & Pr $>|t|$ \\
\hline B-IPQ score & -0.3135 & 0.04224 & $<0.0001$ \\
MMAS-4 score & 1.7239 & 0.4693 & 0.0003 \\
TSQM-9 effectiveness score & 0.06700 & 0.03021 & 0.0270 \\
TSQM-9 convenience score & 0.1275 & 0.03289 & 0.0001 \\
TSQM-9 global satisfaction score & 0.09590 & 0.03270 & 0.0035
\end{tabular}

Only patients with information on the investigated parameters available at both enrollment and 12-month follow-up were included in the model $(N=244)$. CAT and mMRC scores were not included in the model because they were both highly correlated to B-IPQ scores (Spearman's correlation coefficients $>0.65$ and $>0.55$, respectively; $p<0.0001$ in both cases).

measures regression model using DACQ total score $(\geq 80$ vs. $<80 \%)$ as independent variable. The DACQ total score was found to be significantly associated with CAT $(\beta=-2.4420 ; p$ value $=0.0015)$.

\section{Discussion}

COPD poses a major health challenge globally, currently being cause of morbidity, mortality [23], and substantial direct and indirect costs [24]. At the population level, undiagnosis of COPD is common and remained unchanged over 2 decades [25]. Awareness is essential for consciousness and long-term management of chronic diseases, such as COPD. It has been defined as a competency that facilitates self-monitoring [26] and that gives rise to "knowing intentionality" and allows "discerning attitude regarding what to act out and what not" [27]. In other words, awareness goes beyond knowledge. In fact, coping with COPD, patients are confronted by information from external sources (about disease characteristics, causes, prognosis, and treatment) and internal sources (thoughts, emotions, and body sensations) and these are integrated in the complexity of personal experience.

Crucially, to be aware, a patient has not simply to know what COPD is, but also has to recognize the necessity and the role of treatments, to make sense and accept the disease. Our results show that an optimal level of awareness was achieved only for about 1 patient out of 5 . The com- 
parison of patients with DACQ scores $\geq 80$ (optimal awareness level) and patients with DACQ score $<80$ (suboptimal awareness level) showed that they had a different profiles. Patients with suboptimal awareness had a shorter median duration of COPD by 1.4 years and lower treatment satisfaction for the effectiveness and convenience factors and for the global score. Moreover, they had higher B-IPQ scores, indicating a more threatening view of the illness, a worse health status, as testified by CAT, and higher level of dyspnea. In addition, twice as many patients with low COPD awareness, compared to those with good awareness, had one or more exacerbations during the year previous to enrollment. The relationship between COPD awareness and clinical characteristics/PROs during the entire 12-month observation period was explored by repeated measures regression models, as described in the statistical analysis paragraph. The level of awareness was significantly associated with CAT scores, illness perception, adherence, and treatment satisfaction, and the association was in the expected direction. Moreover, during the 12-month observation period, patients with DACQ scores $\geq 80$ had significantly lower CAT scores by 2.4 points on average.

The results of our study seem to show level of awareness is related to a different way to perceive COPD and cope with it and to different clinical outcomes. Awareness seems to be a competency that allows patients to not be passive actors [25] with their disease, but to accommodate changes, to better tolerate their experience, and to increase the positive dimensions of life despite the presence of COPD. To our knowledge, this is the first study that explores the clinical relevance of awareness as a factor that could optimize the long-term management of COPD. Education programs to raise the level of knowledge of patients and their families and to implement recommendations for effective treatment and prevention of COPD are welcome. However, the concept of awareness should take into account also perception, attitudes, and emotions due to their global effect on the ability to adopt strategies for coping with COPD and strengthening of adherence to treatment.

The limitations of our study must be considered. First, patient selection was performed following the inclusion/ exclusion criteria of the SAT study, and the units participating in the study were chosen as a sample of convenience. Moreover, the sample includes only patients who had been referred to tertiary centers. Therefore, in terms of external validity, our study findings should not be generalized to the whole population of patients with COPD, but rather to subjects with similar characteristics than our patient sample. However, it is likely that COPD awareness would have been even lower in a nonspecialist setting. Moreover, it is not possible to assess whether changes of awareness are a direct consequence of changes in clinical outcomes and PROs. We acknowledge that further studies are needed to corroborate the results of our exploratory analysis and to complement them also in more heterogeneous cohorts (including patients with severe COPD features and frequent exacerbations). Nevertheless, our findings could provide input data for developing personalized interventions to improve the level of awareness and to assess their effects on patients' well-being.

\section{Statement of Ethics}

This study protocol of this multicenter, observational perspective cohort study was approved by Ethics Committee of University of Genoa (Comitato Etico Regione Liguria c/o IRCCS A.O.U. San Martino-IST; Protocol N ${ }^{\circ}$ : 343REG2015) and by the institutional Ethics Committees of the centers involved in this study: Comitato Etico della Provincia di Ferrara c/o A.O.U. di Ferrara, Comitato Etico Area Pavia c/o IRCCS Policlinico San Matteo, Comitato Etico Milano Area A c/o A.O. L. Sacco, Comitato Etico Indipendente AOU Policlinico Tor Vergata (PTV), Comitato Etico Centrale Fondazione IRCCS Salvatore Maugeri, Comitato Etico PALERMO 2 c/o A.O. Ospedali Riuniti Villa Sofia Cervello, Segreteria Scientifico-Amministrativa CEAS Umbria, Comitato Etico Area Vasta Nord Ovest c/o A.O.U. Pisana, Comitato Etico Interaziendale A.S.O. S.S. Antonio e Biagio e C. Arrigo, Comitato Etico Regionale delle Marche A.O.U. Ospedali Riuniti, mitato Etico Università degli Studi della Campania AOU Vanvitelli - AORN Ospedali dei Colli, Comitato Etico IRCCS Istituto Tumori "Giovanni Paolo II," Comitato Etico Campania Nord c/o A.O.R.N. Moscati, Comitato Etico della AUSL della Valle D'Aosta, Comitato Etico per le Province di L'Aquila e Teramo c/o ASL Avezzano Sulmona L'Aquila, Comitato Etico Provinciale c/o Azienda Ospedaliera Arcispedale Santa Maria Nuova/I.R.C.C.S., CESC delle Province di Verona e Rovigo c/o AOUI Verona, Comitato Etico Interaziendale A.O. San Luigi Gonzaga, and Comitato Etico Campania Sud c/o ASL Napoli 3 Sud.

\section{Conflict of Interest Statement}

The following authors report personal fees for scientific consultation from Boehringer Ingelheim related to the study: I.B., M.C., A.G.C., N.S., P.R., F.D.M., P.S., F.B., and I.B. report personal fees for scientific consultations and/or lectures at national and international meetings from Boehringer Ingelheim, Chiesi Farmaceutici, GlaxoSmithKline, Menarini, Novartis, Sanofi Genzyme, Biofutura, outside the submitted work. M.C. reports grants for research from AstraZeneca and Chiesi, and reports personal fees for scientific consultations and/or lectures at national and international meetings from Chiesi, Novartis, AstraZeneca, Glaxo Smith 
Kline, Boehringer Ingelheim, Menarini, Zambon, outside the submitted work. A.C.G. has received research funds and speaking or consultancy fees from Astra Zeneca S.P.A.; Boehringer Ingelheim Italia SPA; GlaxoSmithKline S.p.A.; Novartis Farma S.p.A.; Grifols Italia S.P.A.; Stallergenes Italia S.r.l.; Meda Pharma; C.S.L. Behring S.p.A.; A. Menarini Industrie Farmaceutiche Riunite S.r.l.; and Mundipharma Pharmaceuticals S.r.l., Alk Abello' S.P.A. P.R. has participated as a lecturer, speaker, and advisor in scientific meetings and courses under the sponsorship of Almirall, AstraZeneca, Biofutura, Boehringer Ingelheim, Chiesi, GlaxoSmithKline, Menarini Group, Mundipharma, and Novartis. Her department has received funding from Almirall, Boehringer Ingelheim, Chiesi, Novartis, and Zambon. She has no other relevant affiliations or financial involvement with any organization or entity with a financial interest in or financial conflict with the subject matter or materials discussed in the manuscript apart from those disclosed. F.D.M. has received honoraria for lectures at national and international meetings from Almirall, AstraZeneca, Boehringer Ingelheim, Chiesi Farmaceutici, Dompe, Guidotti/Malesci, GlaxoSmithKline, Menarini, Novartis, and Zambon. He has served as a consultant for AstraZeneca, Chiesi Farmaceutici, Novartis, and Zambon and has received financial support for research from Novartis and Boehringer Ingelheim. P.S. reports grants for research from AstraZeneca and Boehringer Ingelheim and reports personal fees for scientific consultations and/or lectures at national and international meetings from Chiesi, Novartis, AstraZeneca, Boehringer Ingelheim, and Zambon, outside the submitted work. F.B. has received honoraria for lectures at national and international meetings from Almirall, AstraZeneca, Boehringer Ingelheim,
Chiesi Farmaceutici, Dompè, Guidotti/Malesci, GlaxoSmithKline, Menarini, Novartis, Lallemand Pharma, Biophutura, Levante Pharma, Merck Sharp and Dohme, and Zambon; he has served as consultant for AstraZeneca, Chiesi Farmaceutici, Novartis, Glaxo Smith Kline, Boehringer Ingelheim, Guidotti/MalesciZambon, Csl Behring. C.S. is employee of Boehringer Ingelheim Italy. F.F. is an employee of MediNeos Observational Research, Modena, Italy. He received payment from Boehringer Ingelheim Italy for the following activities related to the submitted work: scientific support and statistical analysis. N.S. has no conflicts of interest to declare.

\section{Funding Source}

This study was supported by Boehringer Ingelheim S.p.A, Italy.

\section{Author Contributions}

I.B., M.C., A.D.C., P.R., N.S., F.D.M., P.S., C.S., F.F., and F.B. contributed to the design and implementation of the research. F.F. analyzed the data. I.B. and F.B. wrote the paper. All the authors gave final approval of the version to be published and agree to be accountable for all aspects of the work.

\section{References}

1 NIH. National Heart Lung and Blood Institute [webpage on the Internet]. COPD National Action Plan. Available from: https:// www.nhlbi.nih.gov/health-topics/educationand-awareness/COPD-national-action-plan Accessed 2020 July 20.

2 Zielinski J, Bednarek M, Górecka D, Viegi G, Hurd SS, Fukuchi Y, et al. Increasing COPD awareness. Eur Respir J. 2006;27(4):833-52.

3 NIH. National Heart Lung and Blood Institute [webpage on the Internet]. Learn More Breathe Better. Available from: https://www. nhlbi.nih.gov/BreatheBetter Accessed 2020 July 15.

4 BLF. British Lung Fundation [webpage on the Internet]. World COPD Day - 20 November 2019. Available from: https://www.blf.org.uk/ support-for-you/copd/world-copd-day Accessed 2020 June 30.

5 GOLD. Global Initiative for Chronic Obstructive Lung Disease [webpage on the Internet]. World COPD Day - 20 November 2019. Available from: https://goldcopd.org/worldcopd-day/.

6 Boehm A, Pizzini A, Sonnweber T, LoefflerRagg J, Lamina C, Weiss G, et al. Assessing global COPD awareness with Google trends. Eur Respir J. 2019 Jun 27;53(6):1900351.
7 Seo JY, Hwang YI, Mun SY, Kim JH, Kim JH, Park SH, et al. Awareness of COPD in a high risk Korean population. Yonsei Med J. 2015 Mar;56(2):362-7.

8 Mun SY, Hwang YI, Kim JH, Park S, Jang SH, Seo JY, et al. Awareness of chronic obstructive pulmonary disease in current smokers: a nationwide survey. Korean J Intern Med. 2015 Mar;30(2):191-7.

9 Di Marco F, Balbo P, de Blasio F, Cardaci V, Crimi N, Girbino G, et al. Early management of COPD: where are we now and where do we go from here? A Delphi consensus project. Int J Chron Obstruct Pulmon Dis. 2019 Feb 4;14: 353-60.

10 Corli O, Apolone G, Pizzuto M, Cesaris L, Cozzolino A, Orsi L, et al. Illness awareness in terminal cancer patients: an Italian study. Palliat Med. 2009;23(4):354-9.

11 Clare L, Marková IS, Roth I, Morris RG. Awareness in Alzheimer's diseaseand associated dementias: theoretical framework and clinical implications. Aging Mental Health. 2011;15(8):936-44.

12 Morasso G, Alberisio A, Capelli M, Rossi C, Baracco G, Costantini M. Illness awareness in cancer patients: a conceptual framework and a preliminary classification hypothesis. Psychooncology. 1997 Sep;6(3):212-7.
13 Baiardini I, Rogliani P, Santus P, Corsico AG, Contoli M, Scichilone N, et al. Disease awareness in patients with COPD: measurement and extent. Int J Chron Obstruct Pulmon Dis. 2019 Dec 17;14:1-11.

14 Contoli M, Rogliani P, Di Marco F, Braido F, Corsico AG, Amici CA, et al. Satisfaction with chronic obstructive pulmonary disease treatment: results from a multicenter, observational study. Ther Adv Respir Dis. 2019 JanDec;13:1753466619888128.

15 Atkinson MJ, Kumar R, Cappelleri JC, Hass SL. Hierarchical construct validity of the treatment satisfaction questionnaire for medication (TSQM version II) among outpatient pharmacy consumers. Value Health. 2005; 8(Suppl 1):S9-S24.

16 Atkinson MJ, Sinha A, Hass SL, Colman SS, Kumar RN, Brod M, et al. Validation of a general measure of treatment satisfaction, the Treatment Satisfaction Questionnaire for Medication (TSQM), using a national panel study of chronic disease. Health Qual Life Outcomes. $2004 \mathrm{Feb}$ 26;2(2):12.

17 Broadbent E, Petrie KJ, Main J, Weinman J. The brief illness perception questionnaire. J Psychosom Res. 2006;60(6):631-7. 
18 Morisky DE, Green LW, Levine DM. Concurrent and predictive validity of a self-reported measure of medication adherence. Med Care. 1986;24(1):67-74.

19 Jones PW, Harding G, Berry P, Wiklund I, Chen WH, Kline Leidy N. Development and first validation of the COPD assessment test. Eur Respir J. 2009;34(3):648-54.

20 Schilling RS, Hughes JP, Dingwall-Fordyce I. Disagreement between observers in an epidemiological study of respiratory disease. $\mathrm{Br}$ Med J. 1955;1(4905):65-8.

21 Munari AB, Gulart AA, Dos Santos K, Venâncio RS, Karloh M, Mayer AF. Modified medical research council dyspnea scale in GOLD classification better reflects physical activities of daily living. Respir Care. 2018;63(1):77-85.
22 Jaeschke R, Singer J, Guyatt GH. Measurement of health status. Ascertaining the minimal clinically important difference. Control Clin Trials. 1989;10(4):407.

23 GBD 2015 Mortality and Causes of Death Collaborators. Global, regional, and national life expectancy, all-cause mortality, and cause-specific mortality for 249 causes of death, 1980-2015: a systematic analysis for the global burden of disease study 2015 . Lancet. 2016;388(10053):1459-544.

24 Kirsch F, Schramm A, Schwarzkopf L, Lutter JI, Szentes B, Huber M, et al. Direct and indirect costs of COPD progression and its comorbidities in a structured disease management program: results from the LQ-DMP study. Respir Res. 2019 Oct 10;20(1):215.
25 Martinez CH, Mannino DM, Jaimes FA, Curtis JL, Han MK, Hansel NN, et al. Undiagnosed obstructive lung disease in the United States. Associated factors and long-term mortality. Ann Am Thorac Soc. 2015 Dec;12(12): 1788-95.

26 Sedikides C, Skowronski JJ. The symbolic self in evolutionary context. Pers Soc Psychol Rev. 1997;1(1):80-102.

27 Gilbert P. Psychotherapy for the 21st century: an integrative, evolutionary, contextual, biopsychosocial approach. Psychol Psychother. 2019 Jun;92(2):164-89. 